\title{
A preliminary study on the immune responses of HPV16-E7 by combined intranasal immunization with lymphotoxin
}

\author{
Ya-Hong Liu, Hai-Lin Chen, Bo-Qun Xu, Kuang Wei, Xiao-Yan Ying \\ The Second Affiliated Hospital of Nanjing Medical University, Nanjing, China
}

\begin{abstract}
Objectives: Human papillomavirus (HPV) ranks the first cause of cervical cancer. Cervical cancer has high prevalence rates in women around the world. The HPV-E7 oncoprotein is expressed in cervical cancer and is a target of developing immunotherapies against HPV-associated tumors. However, the antigenicity of this protein is low. Due to this reason, potent adjuvants are required to enhance its therapeutic efficacy. This preliminary study aims to evaluate whether lymphotoxin (LT) could act as an effective immune adjuvant for HPV infection in mice models.

Material and methods: Intranasal immunization was used to explore the effect of HPV-E7 and/or LT immune response. After the third intranasal immunization, the titer for the HPV-E7 antibody was detected in serum and vaginal washing fluid. Also, we assessed the expression of chemokine ligand 13 (CXCL13) and Peripheral Node Addressin (PNAd) in the lymph nodes after intranasal immunization with immunohistochemical analysis.

Results: compared to HPV-E7 immunization, intranasal immunization with HPV-E7 plus LT significantly increased HPV-E7-specific serum IgG and vaginal IgA titers. Furthermore, the combined use of HPV-E7 and LT strongly induced E7-specific CTL responses.

Conclusions: LT can be effective for intranasal immunized HPV-E7 to improve E7-specific immune responses to HPV infection. It is new approach to eradicate chronic HPV infection capable of inducing an effective anti-infection method.

Key words: human papillomavirus; HPV-E7; lymphotoxin; intranasal immunization; immunotherapy; lymphocyte homing

Ginekologia Polska 2020; 91, 6: 301-307
\end{abstract}

\section{INTRODUCTION}

Cervical cancer has high prevalence rates in women around the world [1, 2]. According to the World Health Organization (WHO) Human Papillomavirus and Related Cancers, World Summary Report 2010, every year, worldwide, HPV caused 500,000 new cases of cervical cancer [2]. Most HPV infections are harmless and clear up spontaneously; however, persistent HPV infections (especially type 16) have been reported could cause many human cancers such as cervical cancer and oropharyngeal cancer $[3,4]$.

HPV generally encodes six non-structural proteins (E1, E2, E6, E7 E5, E4) and two structural proteins (L1 and L2). These HPV encoded antigens are expressed differentially across the maturing cervical epithelium [1]. It has been reported that natural immune responses to HPV encoded antigens are typically weak and the immune responses vary among individuals, but the E7 protein is an exception to this [5]. The E7 protein is one of the proteins that could elicit a humoral immune response in most cases of invasive cervical cancer [5]. Due to its interactions with regulatory cellular proteins, e.g., p53 and pRb, HPV E7 protein could disrupt the normal cell cycle and the process of DNA repair, leading to neoplastic transformation and the immortalization of epithelial cervical cells. Therefore, E7 is regarded as a powerful transforming oncoprotein target protein for HPV therapeutic strategies. It can directly against HPV infection to prevent and treat the cervical neoplasia [6].

There have been many different attempts to induce E7 specific immune responses in previous studies [7]. For example, Yang et al. [8] conducted a study and found that E7 peptide could induce antigen-specific $T$ cells in animal subjects. However, to date, researchers have not been able to clinically demonstrate an effective therapeutic approach using this method. Several likely reasons exist for this, in- 
cluding the ability of HPV to evade immune recognition [9]. Therefore, there is an urgent need for novel therapies in order to eradicate HPV infection in the genital tract mucous membrane.

Lymphotoxin (LT) is one of the members in tumor necrosis factor (TNF) superfamily that has been shown to be an important component of effector immune responses. LT receptor pathway is essential to protect innate and adaptive immune and LT is essential for host defenses against specific pathogens, for example, it's involved in the production of IgA and many other cytokines [10]. Lymphotoxin (LT) plays multiple roles in the immune system and autoimmunity. It's reported that mucosal immunity is more effective against clear pathogens of mucous membrane infection than traditional immunization like oral administration and injection immunization [7]. Mucosal immunization can be achieved via oral, intranasal, pulmonary, rectal, or vaginal routes, and intranasal immunization with purified HPV16 VLPs induces systemic and mucosal antibody responses [7].Compared to vaginal and rectal mucosal immunity, intranasal immunization is an ideal method to induce genital mucosal immune response because it is cost-effective and convenient [11, 12].

In consideration of the above, we conducted this preliminary study to explore whether co-administration of E7 peptides and LT with the addition of cholera toxin (CT) elicits immune responses as well as protects against the tumor. Our results showed that intranasal co-administration of E7 peptides and LT induced stronger antigen-specific immune responses in mice when compared to the response from E7 alone. Further, LT stimulation significantly enhanced E7-specific T-cell responses and CTL responses. We also demonstrated that co-administration of E7 peptides with LT could induce lymphocyte homing in the mouse models.

\section{MATERIAL AND METHODS}

\section{Animals}

All C57BL/6 mice (female, 6-8 weeks old) were purchased from Animal Center of Nanjing Medical University (Nanjing, China). Animals in this study were kept in the pathogen-free conditions. All animal care and experimental procedures were performed in accordance with recommendations for the proper use and care of laboratory animals.

The study was approved by the Ethics Committee of our hospital.

\section{Recombinant E7 peptide and LT}

Recombinant peptide of HPV-16E7 protein (amino acids: 31-50 SSEEEDEIDGPAGQ AEPDRA) was synthesized by the GL Biochem (Shanghai, China). The peptide purity was above $98 \%$ as determined by reverse-phase high-performance liquid chromatography. E7 peptide was dissolved in double-distilled $\mathrm{H}_{2} \mathrm{O}$. All peptides that were not immediately used were stored at $-20^{\circ} \mathrm{C}$. LT with a purity of $>98 \%$. was purchased from PeproTech (NJ, USA).

\section{Immunization of mice}

Twenty-four mice were randomly assigned to four groups ( $n=6$ each): PBS control group, LT group, HPV-E7 group and HPV-E7 plus LT group. The mice in the four groups were lightly anesthetized with $10 \%$ ketamine and $5 \%$ xylazine in sterile water for the duration of the inoculation. The mice in HPV-E7 + LT group were immunized intranasally with $10 \mu \mathrm{g}$ of HPV-E7 and $2 \mu \mathrm{g}$ of cholera toxin (Sigma- Aldrich, MO, USA) and injected through the vena caudalis with $0.05 \mu \mathrm{g}$ of Lymphotoxin in a total volume of $20 \mu \mathrm{L}$ per mouse. The mice in HPV-E7 group were immunized intranasally with $10 \mu \mathrm{g}$ of HPV-E7 and $2 \mu \mathrm{g}$ of cholera toxin alone. The mice in the LT group received $0.05 \mu \mathrm{g}$ of Lymphotoxin in a total volume of $20 \mu \mathrm{L}$ per mouse and the PBS control group received PBS only. All mice in the four groups were immunized three times with the same dosage of the corresponding immune formulation at two-week intervals. The mice in control group were given PBS in the same volume on days 0,14 , and 28 .

\section{Collection of vaginal samples and serum}

Samples of blood and vaginal were collected on day 0 , 14,28 , and 42 following intranasal immunization and/or LT injection. Blood samples were obtained by retro-orbital sinus puncture and clotted at room temperature for $60 \mathrm{~min}$ utes. After microcentrifuged at 1,700 $\mathrm{g}$ for 10 minutes, the serum was separated and then stored immediately at $-20^{\circ} \mathrm{C}$ until analysis. In order to obtain the vaginal samples, $100 \mu \mathrm{L}$ PBS were injected into the vaginal cavity. The vaginal samples were then washed five times in the lab.

\section{Antibody Detection}

HPV-E7 antibody titers were detected using enzyme-linked immunosorbent assay at dilutions of 100 , $200,400,800,1600,3200$ for detections of IgG, and at delusions of 10, 20, 40 for detections of IgA. For detection of HPV-E7-specific vaginal $\lg A$ and serum $\lg G$, high protein-binding 96-well plates (Invitrogen, Burlington, ON, Canada) were incubated overnight at $4^{\circ} \mathrm{C}$, with a capture layer of $100 \mu \mathrm{L}$ HPV-E7 peptide $(50 \mu \mathrm{g} / \mathrm{mL}$ in carbonate buffer, pH 9.6). After three washes with PBS-0.05\% Tween-20, the plates were blocked with $3 \%$ bovine serum albumin in PBS at room temperature for 2 to 4 hours, then samples were applied in duplicate at various dilutions in $3 \%$ bovine serum albumin-PBS as indicated in individual experiments, and incubated at $4^{\circ} \mathrm{C}$ overnight. The plates were washed five times, incubated with peroxidase-conjugated goat anti-mouse IgA (Santa Cruz, CA, USA), diluted 1:5000 in 1\% bovine serum albumin-PBS or anti-mouse IgG (Santa Cruz, CA, USA), then diluted 1:2500, at room temperature for 1 hour. 
After seven washes, $100 \mu \mathrm{L}$ of TMB (Sigma Aldrich, MO, USA) were added to each well. Colorimetric reactions were performed at room temperature in the dark then stopped by adding $1 \mathrm{~N} \mathrm{H}_{2} \mathrm{SO}_{4}$. ODs were measured at $450 \mathrm{~nm}$ using a 96-well spectrophotometer (Microplate Reader Model 680, BIO-RAD, USA). End point titers were determined and expressed as geometric mean titers. The mean background optical density value of non-immune mice was measured twice and used as the cutoff for determining positive values.

\section{DC preparation}

Spleen DC was obtained aseptically from C57BL/6 mice and placed in the PBS buffer. After dissociation, suspension was collected through a 400-mesh steel net. First, red blood cells were removed by adding ACK solution (Biofluids, Camarillo, CA) and the remaining cells were resuspended with RPMI 1640 medium containing 10\% fetal bovine serum, then counted. Non-adherent cells were disposed of and the remaining cells were divided into two parts. Spleen cells were labeled with $2.5 \mu \mathrm{mol} / \mathrm{L}$ Carboxy Fluoroscein Succinimidyl Ester (5- or 6-(N- uccinimidyloxycarbonyl)-3,6'-0,0'diacetyl-fluorescein, CFSE; Invitrogen, USA) (CFSE high) or $0.25 \mu \mathrm{mol} / \mathrm{L}$ CFSE (CFSE low). CFSE high cells were then pulsed for $90 \mathrm{~min}$ at $37^{\circ} \mathrm{C}$ with either $2 \mu \mathrm{g} / \mathrm{mL}$ HPV-E7 or a survivin peptide as a control in a $5 \% \mathrm{CO}_{2}$ incubator. After extensive washing, CFSE-high and low cells were mixed in a 1:1 ratio.

\section{Flow cytometric analysis}

After being stimulated three times, the CFSE-high and CFSF-low cells $\left(2 \times 10^{6}, 100 \mu \mathrm{L}\right)$ were mixed in a ratio of 1:1 and injected intravenously or vaginally under light anesthesia. After 12 hours of administration, the spleen and uterus were processed into single cell suspensions. Briefly, after perfusion with cold $1 \mathrm{x}$ PBS, the spleen was removed, minced into fragments, and digested with $1 \mathrm{mg} / \mathrm{mL}$ collagenase (catalog no. 17018-029; Gibco) and $0.1 \mathrm{mg} / \mathrm{mL}$ DNAase for 1 hour at $37^{\circ} \mathrm{C}$ with intermittent agitation. Spleen fragments were passed through a 400mesh steel net (Falcon; BD Biosciences). and approximately 13-107 cells were incubated in $2.5 \mathrm{mg} / \mathrm{mL}$ Fc blocking solution, centrifuged $\left(800 \times \mathrm{g}, 10\right.$ minutes, $\left.8^{\circ} \mathrm{C}\right)$, and resuspended with FACS buffer.

Approximately $1 \times 10^{5}$ to $10^{6}$ cells per sample were measured to assess the clearance rate of the target cells. The following formula was used: the specific killing rate $(\%)=[1-($ CFSE-low control/CFSE-high control)/(CFSE-low experimental/CFSE-high experimental)] $\times 100 \%$. The formula was used to measure the percent of cells that were killed. Data were acquired on a Becton Dickinson FACSCalibur flow cytometer and analyzed with FlowJo software (Tree Star Inc., Ashland, OR, USA).

\section{Statistical analysis}

All data are expressed as mean \pm SD. Graphpad Prism (version 5; San Diego, USA) was used to construct figures and diagrams. One-way analysis of variance (ANOVA) or unpaired T-tests and Mann-Whitney tests were used where appropriate. Differences were considered statistically significant if the $p$-value was less than 0.05 . Error bars represent \pm SD.

\section{RESULTS}

HPV-E7 plus LT enhances E7-specific systemic humoral immune response

To evaluate the adjuvant properties of LT in vivo, the geometric mean titers of the antigen-specific antibody were analyzed in this study. At two-week intervals, mice were administrated three times with the indicated amount of HPV-E7 plus LT, HPV-E7, LT, or PBS. In addition, CT was added in each group as a mucosal adjuvant. For 42 days, serum samples were collected after intranasal immunization and titrated for HPV-E7-specific antibodies. Animals immunized with HPV-E7 showed detectable HPV-E7-specific antibodies. HPV-E7 plus LT elicited a significantly higher increase of HPV-E7-specific antibody levels compared to inoculation with HPV-E7 alone. However, the mice inoculated with LT alone did not show any increase in humoral immune response (Fig. 1). These results indicated that LT could significantly enhance the HPV-E7 peptide-specific humoral immune response.

\section{HPV-E7 plus LT enhances E7-specific vaginal humoral immune response}

We also examined the adjuvant properties of LT in the HPV-E7-specific vaginal humoral immune response. The level of vaginal IgA was 1:40 in the HPV-E7 plus LT group and 1:20 in the HPV-E7 group (Fig. 2). At the dilution level of 1:20 and 1:40, the HPV-E7-specific antibody titers were higher in the HPV-E7 plus LT group than those in the HPV-E7 group. Our results demonstrated that a significant

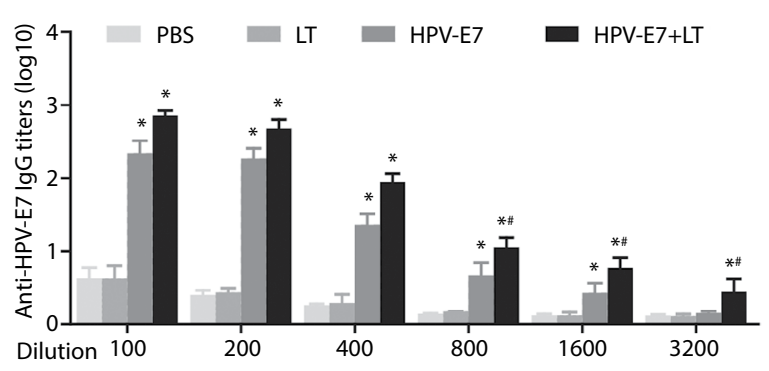

Figure 1. Antibody response to HPV-E7 and/or LT in vivo. Mice were immunized with PBS, HPV-E7 and/or LT at two-week intervals. Serum was collected 42 days after immunization and diluted into different concentration. IgG responses against HPV-E7 were evaluated using ELISA; ${ }^{*} p<0.05$ as compared to PBS control mice; ${ }^{*} p<0.05$ as compared to HPV-E7 naive mice 


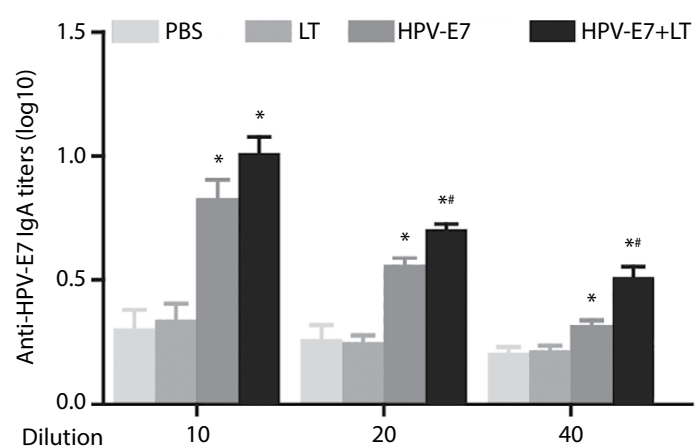

Figure 2. HPV E7-specific vaginal humoral immune response to HPV-E7 and/or LT in vivo. C57BL/6 mice were immunized with HPV-E7 and/or LT at two-week intervals. Vaginal washes were collected after HPV-E7 and/or LT immunization for 42 days and diluted into different concentrations. HPV-E7-specific IgA antibodies were evaluated using ELISA; ${ }^{*} \mathrm{p}<0.05$ as compared to PBS control mice; ${ }^{*} \mathrm{p}<0.05$ as compared to HPV-E7 naive mice
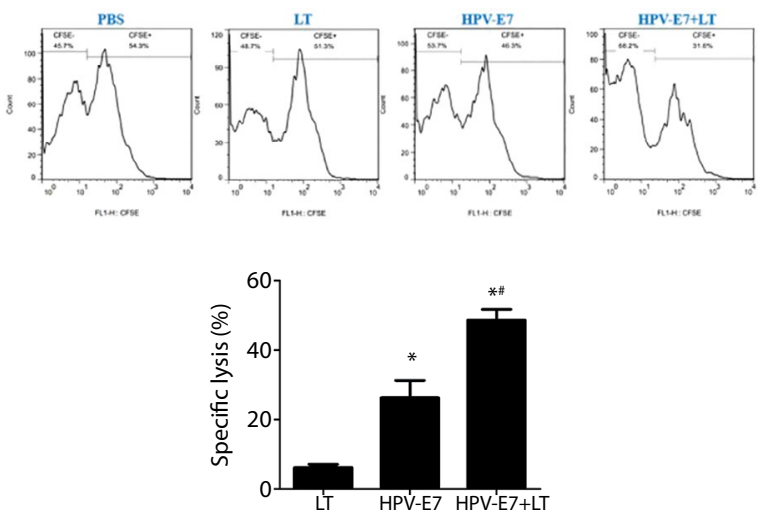

Figure 3. Mice were immunized with PBS, HPV-E7 and/or LT at two-week intervals then injected with syngeneic CFSE-labeled splenocytes pulsed with E7 peptide to measure spontaneous cytotoxicity generated by CTL cells. The peptide-specific killing response in vivo was determined $7 \mathrm{~d}$ later. The killing rate of CTL was calculated using the following formula: the specific killing rate (\%) = [1 - (CFSE-low control/CFSE-high control)/(CFSE-low experimental/ CFSE-high experimental)] $\times 100 \%$; $\mathrm{p}<0.05$ as compared to PBS control mice; $\# p<0.05$ as compared to HPV-E7 naive mice

HPV-E7-specific vaginal humoral immune response was induced by LT.

\section{Immunization with HPV-E7 peptide plus LT stimulated enhanced systemic anti-HPV-E7 CTL responses}

We investigated whether HPV-E7 + LT immunization was able to induce anti-HPV-E7 CTL responses. The mice were immunized three times with HPV-E7 + LT, HPV-E7or PBS. The splenocytes of the immunized mice were stimulated with HPV-E7 and cytometric analysis was used to detect the activities of the target cells killed by HPV-E7-specific CTL. M1 represented CFSF-low cells and M2 represented
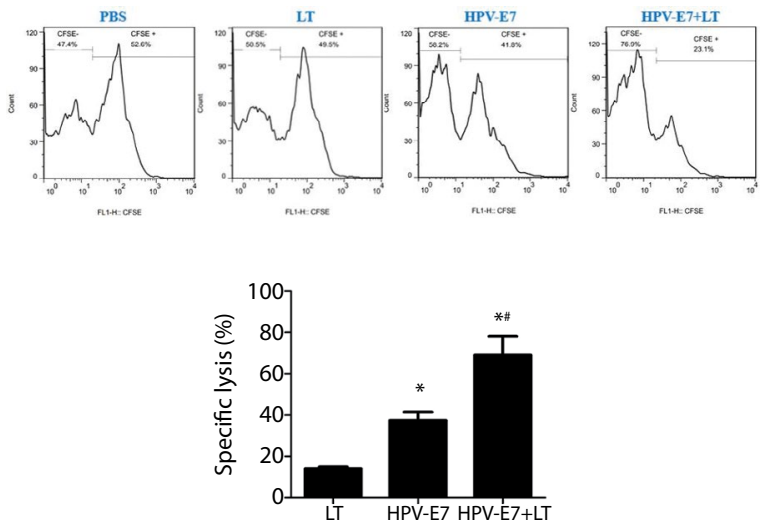

Figure 4. Mice were immunized with PBS, HPV-E7, and/or LT then injected with syngeneic CFSE-labeled splenocytes pulsed with E7 peptide to measure vaginal specific cytotoxicity generated by CTL cells. HPV-E7-specific killing response in vaginal washes was determined $7 \mathrm{~d}$ later; ${ }^{*} \mathrm{p}<0.05$ as compared to PBS control mice; \# $\mathrm{p}<0.05$ as compared to HPV-E7 naive mice

CFSE-high cells (Fig. 3). M1 and M2 cells in the control group were substantially equal, there were no obvious cytotoxicities of CTL or killing effects for target cells incubated with HPV-E7. There were fewer M2 cells than M1 cells in both the HPV-E7 and HPV-E7 + LT groups. This means that immunization with HPV-E7 plus LT induced a significant increase of the HPV-E7-specific killing response in vivo. The killing rate of HPV-E7 + LT group was higher than that in HPV-E7 alone group $(51.64 \% \pm 3.07 \%$ vs $26.25 \% \pm 5.04 \%, p<0.05)$. And the killing rate of the PBS group was $(6.16 \% \pm 0.97 \%)$.

\section{HPV-E7 peptide plus LT stimulated enhanced E7- specific vaginal CTL responses}

To determine whether LT modulates a vaginal HPV-E7-specific response, vaginal samples were obtained and HPV-E7-specific IgA were examined. We found that the killing rates of the HPV-E7 + LT group increased significantly compared to HPV-E7 immunization alone $(69.12 \% \pm 9.01 \%$ vs $37.46 \pm 4.03 \%, p<0.05)$. Our results suggested that LT played an important role in the effect of CTL-mediated cytotoxicities (Fig. 4).

\section{HPV-E7 peptide plus LT induces lymph homing}

In the present study, we assessed the capacity of protective T-cell subpopulations to home to the genital mucosa and associated lymphoid tissues in response to HPV-E7 and LT immunization. Tissue section morphometry of lymph nodes showed the mice that were immunized with HPV-E7 + LT had the most abundant CXCL13 and PNAd levels. The mice that were immunized with either PBS or LT alone had the lowest CXCL13 and PNAd levels. There were no differences in the PBS and LT immunization positive 


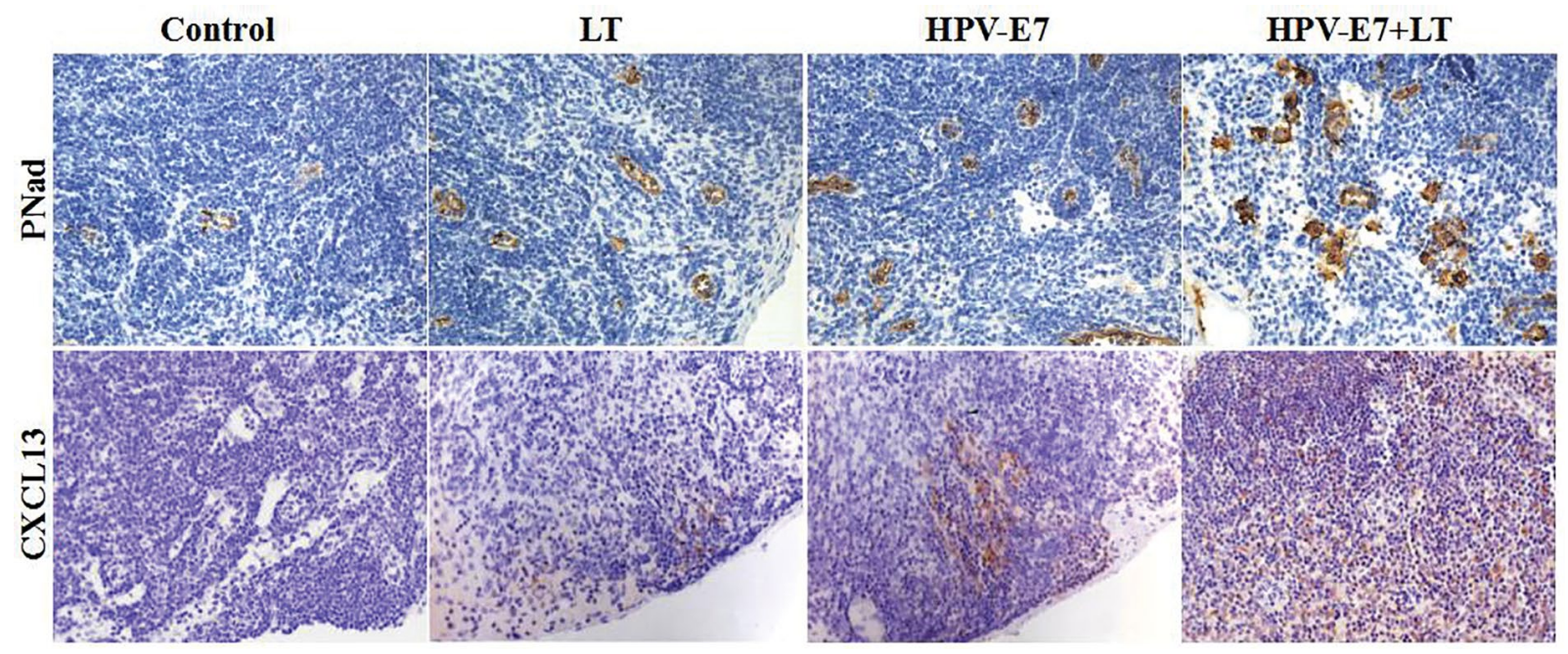

Figure 5. Mice were immunized with PBS, HPV-E7 and/or LT at two-week intervals. Representative images for the immunohistochemical staining of PNAd and CXCL13 in lymph nodes of different groups (400X)

areas regarding CXCL13 and PNAd. These results demonstrate that HPV-E7 immunization contributes to lymphocyte homing to genital mucosa. Our findings that lymph nodes with HPV-E7+LT immunization express stronger lymphocyte "homing" chemokines CXCL13 and PNAd than those immunized with HPV-E7 alone, suggest that LT is critical to promoting lymphocyte homing induced by HPV-E7 immunization (Fig. 5).

\section{DISCUSSION}

Each year, worldwide, there are approximately 529,000 newly diagnosed cases of cervical cancer, which have approximately a $50 \%$ mortality rate [13]. In the occurrence of cervical cancer, HPV plays an important role. Traditional methods to manage cervical cancer are focused on resolving the tumor. The methods to treat viral infection are usually neglected. Therefore, recurrence of cervical cancer often happens after successful treatment. To effectively eliminate HPV precancerous lesions and eliminate recurrence, therapeutic treatments for HPV infection are required. It has been reported that peptide-based immunotherapy is promising for resolving both cancer and HPV infection, but its full potential has yet to be investigated and its inherent limitations have not been overcome [14]. High-risk HPV-E7 is generally known as the most important oncogene of cervical cancer [6]. E7 is an HPV protein that is expressed during early phases of viral infections. Data indicates that E7 is capable of activating the specific CTL cells which act as effector cells to eliminate HPV infected cells by directly killing or releasing tumor inhibitive factors [15]. In this study we tested HPV-E7 as a target for an effective immune therapy and immune regulatory factors to eliminate HPV infection in the genital tract. It was difficult to induce intense im- munoreactions and clear pathogens adhering to mucous surface using conventional subcutaneous immunization in genital mucosa. We chose to deliver HPV-E7 via the nasal route because this route had higher mucosal antibody titers than the other mucosal routes of immunization (oral, rectal, and vaginal) [15]. Also, we use CT to enhance HPV-E7-specific antibody response.

\section{Intranasal immunization with HPV-E7 peptide} stimulated enhanced E7-specific systemic and vaginal humoral and cellular immune responses

Most HPV infections begin at the mucosal surfaces; therefore, immunity against HPV infection might depend on the mucosal immune response [16]. This makes the mucosal route the most appropriate way for immunization due to its ability to induce both mucosal and systemic immune responses [16]. Mucosal immunity is a special part of the immune system and is associated with most lymphoid tissues (> 50\%) characterized by common mucosal effects, including gut, nasal and urogenital associated lymphoid tissues [17]. Plasma cells and sensitized lymphocytes activated in inductive sites home to diffuse lymphoid tissues of various mucosae through interaction with lymphocyte homing receptors, endothelial cell adhesion molecules, and corresponding peripheral node addressins to induce immune response. Humoral and cell-mediated antigen immune responses can be induced by mucosal immunization. Mucosal immunization can be achieved via oral, intranasal, pulmonary, rectal, or vaginal routes [18-19]. It has been reported that intranasal immunization with purified HPV16 VLPs induces systemic and mucosal antibody responses [20]. Therefore, we used intranasal immunization to explore the effect of HPV-E7 and/or LT immune response. 
Also, CT was used as a mucosal adjuvant to enhance mucosal antibody response.

Two weeks after the third intranasal immunization in C57BL/6 mice, the titer for the HPV-E7 specific antibody was detected in the serum and vaginal washing fluid, following CT treatment. Our results showed that the titers for the HPV-E7 specific antibody in the serum and vaginal washing fluid were higher than in the control group following administration of CT, which suggested that appropriate humoral immunity responses were generated after HPV-E7 intranasal immunization.

LT is one of the members in the TNF superfamily and was originally identified as a lymphocyte product that was able to exert cytotoxic effects on tumor cells in vitro [10,21]. Previous studies have found that LT- $a$ and its complex play crucial roles in nasal-associated lymphoid tissue development and function [22]. The cytokine LT is a promising candidate for use in cancer therapy. It is able to kill various specific cancer cells. Therefore, LT has received increased public attention and is considered a promising antitumor and antivirus biological agent.

In our study, we showed that HPV-E7, with the help of an LT adjuvant, following CT treatment, can elicit a significant HPV-E7-specific response in the immune system and vaginal tract. We also found that HPV-E7 plus LT can elicit a strong E7-specific immune response in vivo, as evidenced by a significantly enhanced systemic and vaginal humoral immune response, cytotoxicity, and lymph homing, compared with control groups. The data presented here demonstrated that LT markedly promoted humoral and cellular responses of the immune system and genital tract mucosa after intranasal immunization with HPV-E7 following administration of CT. LT can effectively strengthen the clearance rate of HPV infected cells in the local genital tract by HPV-E7. It should be noted that the differences between HPV-E7 plus LT and HPV-E7 were only significant at high dilutions, which needs further explorations in future studies and also needs improved to achieve a goal that at a much lower dilution, HPV-E7 plus LT can demonstrate a significant effect.

Hydrogen-bonded multilayered Tannic Acid (TA), a novel nanothin polymer material, showed similar effects to LT in anticancer immunotherapy [23]. TA was designed through hydrogen-bonded interactions of a natural polyphenol with poly (N-vinylpyrrolidone) (PVPON) deposited via layer-by-layer (LbL) assembly. The combination of TA and HPV-E7 with LT will be considered in future research [24].

\section{LT stimulating HPV-E7 intranasal immunization by inducing lymph homing}

The mucosal immune system is the first line to defense against pathogen invasion. Different parts of the mucosa can be connected by lymphocyte homing, generating a common immunological effect. The lymphocyte homing process is related to a variety of migrated molecule cascades that are involved in the lymphocyte homing process. These include lymphocyte homing receptors, addressins, and lymphatic chemotactic factors and their receptors. L-selectin, one of the lymphocyte homing receptors, is a member of the cellular adhesion molecular selectin family that participates in the process of lymphocyte homing and recycling [25]. PNAd is the ligand of L-selectin. It is first obtained from high endothelial venules of mice lymphocytes by monoclonal antibody MECA-79 and over-expressed in nasal-associated lymphoid tissue [26]. Lymphatic chemotactic factor CXCL13, also known as B-cell chemotactic factor, is a member of CXC chemotactic factor family. CXCL13 can induce the migration and homing of mature $B$ lymphocytes after integrating with its receptors.

This preliminary study found that adhesion molecules, lymphatic chemotactic factors, and addressins were low-expressed or non-expressed in LT knockout mice [22]. In this study, we used the lymphocyte homing related factors CXCL13 and PNAd to explore the possible target factors adjusted by LT in the lymphocyte homing pathway after intranasal immunization with HPV-E7. We found that the expression of CXCL13 and PNAd was elevated significantly in lymph nodes with HPV-E7 + LT immunization compared to those immunized with HPV-E7 alone. This demonstrates that $L T$ regulates the immunological effects of intranasal immunization by stimulating activation and homing of lymphocytes.

The immunogen used in this study is a recombinant peptide of HPV-16 E7 protein, which may not be able to protect against other subtypes of HPV virus. In addition, intranasal vaccination may not be so effective as intravaginal vaccination, but its advantage is that it's convenient and has lower risks of infections.

In conclusion, specific humoral and cellular immune responses of the immune system and genital tract can be induced by intranasal administration of HPV-E7. LT can effectively promote the effects of intranasal immunization via regulating expression of lymphocyte homing related chemokines in the presence of CT. Further study is required to fully understand the detailed mechanism of regulating the homing factors of LT.

\section{REFERENCES}

1. Cohen P, Jhingran A, Oaknin A, et al. Cervical cancer. The Lancet. 2019; 393(10167): 169-182, doi: 10.1016/s0140-6736(18)32470-x.

2. World Health Organization (WHO). Human Papillomavirus and Related Cancers. SummaryReport Update. 2010.

3. de Sanjosé S, Brotons M, Pavón MA. The natural history of human papillomavirus infection. Best Pract Res Clin Obstet Gynaecol. 2018; 47: 2-13, doi: 10.1016/j.bpobgyn.2017.08.015, indexed in Pubmed: 28964706.

4. Li Y, Xu C. Human Papillomavirus-Related Cancers. Adv Exp Med Biol. 2017; 1018: 23-34, doi: 10.1007/978-981-10-5765-6 3, indexed in Pubmed: 29052130 . 
5. Banister CE, Liu C, Pirisi L, et al. Identification and characterization of HPV-independent cervical cancers. Oncotarget. 2017; 8(8): 13375-13386, doi: 10.18632/oncotarget.14533, indexed in Pubmed: 28077784.

6. Mittal S, Banks L. Molecular mechanisms underlying human papillomavirus E6 and E7 oncoprotein-induced cell transformation. Mutat Res Rev Mutat Res. 2017; 772: 23-35, doi: 10.1016/j.mrrev.2016.08.001, indexed in Pubmed: 28528687.

7. Dorta-Estremera S, Chin RL, Sierra G, et al. Mucosal HPV E6/E7 Peptide Vaccination in Combination with Immune Checkpoint Modulation Induces Regression of HPV Oral Cancers. Cancer Res. 2018; 78(18): 5327-5339, doi: 10.1158/0008-5472.CAN-18-0892, indexed in Pubmed: 30054333.

8. Yang $Y, C h e Y$, Zhao $Y$, et al. Prevention and treatment of cervical cancer by a single administration of human papillomavirus peptide vaccine with CpG oligodeoxynucleotides as an adjuvant in vivo. Int Immunopharmacol. 2019;69: 279-288, doi: 10.1016/j.intimp.2019.01.024, indexed in Pubmed: 30743204.

9. Einstein $\mathrm{MH}$, Schiller JT, Viscidi RP, et al. Clinician's guide to human papillomavirus immunology: knowns and unknowns. Lancet Infect Dis. 2009; 9(6): 347-356, doi: 10.1016/S1473-3099(09)70108-2, indexed in Pubmed: 19467474.

10. Upadhyay V, Fu YX. Lymphotoxin signalling in immune homeostasis and the control of microorganisms. Nat Rev Immunol. 2013; 13(4): 270-279, doi: 10.1038/nri3406, indexed in Pubmed: 23524463.

11. Kozlowski PA, Williams SB, Lynch RM, et al. Differential induction of mucosal and systemic antibody responses in women after nasal, rectal, or vaginal immunization: influence of the menstrual cycle. J Immunol. 2002; 169(1): 566-574, doi: 10.4049/jimmunol.169.1.566, indexed in Pubmed: 12077289.

12. Johansson EL, Wassén $L$, Holmgren J, et al. Nasal and vaginal vaccinations have differential effects on antibody responses in vaginal and cervical secretions in humans. Infect Immun. 2001; 69(12): 7481-7486, doi: 10.1128/IAI.69.12.7481-7486.2001, indexed in Pubmed: 11705923.

13. Bray F, Ferlay J, Soerjomataram I, et al. Global cancer statistics. CA Cancer J Clin. 2011; 61(2): 69-90, doi: 10.3322/caac.20107, indexed in Pubmed: 21296855.

14. van der Burg SH, Melief CJM. Therapeutic vaccination against human papilloma virus induced malignancies. Curr Opin Immunol. 2011; 23(2): 252-257, doi: 10.1016/j.coi.2010.12.010, indexed in Pubmed: 21237632 .
15. Eberl G. A new vision of immunity: homeostasis of the superorganism Mucosal Immunol. 2010;3(5): 450-460, doi: 10.1038/mi.2010.20, indexed in Pubmed: 20445502.

16. Gardella B, lacobone AD, Musacchi V, et al. The Mucosal Innate Immune Response in Primary Human Papillomavirus Infection: A Pilot Study. J Low Genit Tract Dis. 2016; 20(4): 338-342, doi: 10.1097/LGT.0000000000000245, indexed in Pubmed: 27490077.

17. Yuki Y, Kiyono H. New generation of mucosal adjuvants for the induction of protective immunity. Rev Med Virol. 2003; 13(5): 293-310, doi: 10.1002/rmv.398, indexed in Pubmed: 12931340.

18. Longet $\mathrm{S}$, Lundahl MLE, Lavelle EdC. Targeted Strategies for Mucosal Vaccination. Bioconjug Chem. 2018; 29(3): 613-623, doi: 10.1021/acs. bioconjchem.7b00738, indexed in Pubmed: 29300463.

19. Bernocchi $B$, Carpentier R, Betbeder D. Nasal nanovaccines. Int J Pharm. 2017; 530(1-2): 128-138, doi: 10.1016/j.ijpharm.2017.07.012, indexed in Pubmed: 28698066.

20. Nardelli-Haefliger D, Lurati F, Wirthner D, et al. Immune responses induced by lower airway mucosal immunisation with a human papillomavirus type 16 virus-like particle vaccine. Vaccine. 2005; 23(28): 3634-3641, doi: 10.1016/j.vaccine.2005.02.019, indexed in Pubmed: 15882523.

21. Koroleva EP, FuYX, Tumanov AV. Lymphotoxin in physiology of lymphoid tissues - Implication for antiviral defense. Cytokine. 2018; 101:39-47, doi: 10.1016/j.cyto.2016.08.018, indexed in Pubmed: 27623349.

22. Ying $X$, Chan $K$, Shenoy $P$, et al. Lymphotoxin Plays a Crucial Role in the Development and Function of Nasal-Associated Lymphoid Tissue through Regulation of Chemokines and Peripheral Node Addressin. Am J Pathol. 2005; 166(1): 135-146, doi: 10.1016/s0002-9440(10)62239-0.

23. Liu F, Kozlovskaya V, Zavgorodnya O, et al. Encapsulation of anticancer drug by hydrogen-bonded multilayers of tannic acid. Soft Matter. 2014; 10(46): 9237-9247, doi: 10.1039/c4sm01813c, indexed in Pubmed: 25284271.

24. Kozlovskaya V, Xue B, Lei W, et al. Hydrogen-bonded multilayers of tannic acid as mediators of T-cell immunity. Adv Healthc Mater. 2015; 4(5): 686-694, doi: 10.1002/adhm.201400657, indexed in Pubmed: 25491369.

25. Rosen SD. Ligands for L-selectin: homing, inflammation, and beyond. Annu Rev Immunol. 2004; 22: 129-156, doi: 10.1146/annurev.immunol.21.090501.080131, indexed in Pubmed: 15032576.

26. Hemmerich S, Butcher EC, Rosen SD. Sulfation-dependent recognition of high endothelial venules (HEV)-ligands by L-selectin and MECA 79, and adhesion-blocking monoclonal antibody. J Exp Med. 1994; 180(6): 2219-2226, doi: 10.1084/jem.180.6.2219, indexed in Pubmed: 7525849. 